\title{
Unitarity Triangle global fits testing the Standard Model: UTfit 2021 Standard Model update
}

\author{
Marcella Bona, ${ }^{a, *}$ Marco Ciuchini, ${ }^{b}$ Denis Derkach, ${ }^{c}$ Fabio Ferrari, ${ }^{d}$ Enrico Franco, ${ }^{e}$ \\ Vittorio Lubicz, ${ }^{f}$ Guido Martinelli, ${ }^{g}$ Maurizio Pierini, ${ }^{h}$ Luca Silvestrini, ${ }^{i}$ Cecilia \\ Tarantino, ${ }^{f}$ Vincenzo Vagnoni, ${ }^{j}$ Mauro Valli $^{k}$ and Ludovico Vittorio ${ }^{l}$ \\ ${ }^{a}$ Queen Mary University of London \\ ${ }^{b}$ INFN Sezione di Roma Tre \\ ${ }^{c}$ Yandex/Higher School of Economics \\ ${ }^{d}$ University of Bologna and INFN Sezione di Bologna \\ ${ }^{e}$ INFN Sezione di Roma \\ ${ }^{f}$ University of Roma Tre \\ ${ }^{g}$ University of Roma La Sapienza \\ ${ }^{h}$ CERN \\ ${ }^{i}$ INFN Sezione di Roma \\ ${ }^{j}$ INFN Sezione di Bologna \\ ${ }^{k}$ Stony Brook University \\ ${ }^{l}$ Scuola normale Superiore, Pisa and INFN Sezione di Pisa
}

Flavour physics represents a unique test bench for the Standard Model (SM). New analyses performed at the LHC experiments are now providing unprecedented insights into CKM metrology and new evidences for rare decays. The CKM picture can provide very precise SM predictions through global analyses. We present here the results of the latest global SM analysis performed by the UTfit collaboration including all the most updated inputs from experiments, lattice QCD and phenomenological calculations.

\footnotetext{
*** The European Physical Society Conference on High Energy Physics (EPS-HEP2021), ***

*** 26-30 July $2021 * * *$

*** Online conference, jointly organized by Universität Hamburg and the research center DESY ***
}

\footnotetext{
*Speaker
} 
Flavour physics can test the Standard Model (SM) with high precision to quantify the coherence of its picture and to explore possible departures from it. Performing a global fit of flavour results, we can extract the most accurate determination of the parameters of the Cabibbo-Kobayashi-Maskawa (CKM) matrix [1], as well as the best SM predictions for a wide range of flavour observables. The Unitarity Triangle (UT) analysis here presented is performed by the UT $f$ it Collaboration following the method described in Refs. [2]. We updated the analysis with the latest determinations of the theoretical inputs and the latest measurements of the experimental observables. The basic constraints used in the global fit and contributing to the sensitivity of the CKM matrix elements are: $\left|V_{u b} / V_{c b}\right|$ from semileptonic $B$ decays, $\Delta m_{d}$ and $\Delta m_{s}$ from $B_{d, s}^{0}$ oscillations, $\varepsilon_{K}$ from neutral $K$ mixing, $\alpha$ UT angle from charmless hadronic $B$ decays, $\gamma$ UT angle from charm hadronic $B$ decays, and the sine of $2 \beta$ UT angle from $B^{0} \rightarrow J / \psi K^{0}$ decays.

The values of most experimental inputs are taken from the Heavy Flavour Averaging Group (HFLAV) (in Ref. [3] and online update at the hflav. web. cern. ch, mostly for the Particle Data Group 2021 update [4]). However, in the cases detailed in the text below, the UTfit collaboration performs its own averages. On the theoretical side, the non-perturbative QCD parameters are mostly taken from the recent lattice QCD determinations as in Ref. [5]. The continuously updated set of numerical values used as inputs can be found at www.utfit.org.

\section{Updated inputs and results of the global fit in the Standard Model}

The full list of measurements used as inputs in the global fit is given in the first and second columns of Table 1.

Table 1: Full SM inputs with their predictions from the SM global fits.

\begin{tabular}{lccl}
\hline \hline Input & Measurement & Fit prediction & Pull \\
\hline $\sin 2 \beta$ & $0.688 \pm 0.020$ & $0.750 \pm 0.027$ & $\sim 1.8$ \\
$\gamma$ & $66.1 \pm 3.5$ & $66.1 \pm 2.1$ & $<1$ \\
$\alpha$ & $93.6 \pm 4.2$ & $90.5 \pm 2.1$ & $<1$ \\
$\varepsilon_{K} \cdot 10^{3}$ & $2.228 \pm 0.001$ & $2.00 \pm 0.14$ & $\sim 1.5$ \\
\hline$\left|V_{c b}\right| \cdot 10^{3}$ & $41.1 \pm 1.0$ & $41.9 \pm 0.5$ & $<1$ \\
$-\left|V_{c b}\right| \cdot 10^{3}(\mathrm{excl})$ & $39.09 \pm 0.68$ & & $\sim 3.6$ \\
$\left|V_{c b}\right| \cdot 10^{3}(\mathrm{incl})$ & $42.16 \pm 0.50$ & & $<1$ \\
\hline$\left|V_{u b}\right| \cdot 10^{3}$ & $3.89 \pm 0.21$ & $3.68 \pm 0.10$ & $<1$ \\
$-\left|V_{u b}\right| \cdot 10^{3}(\mathrm{excl})$ & $3.73 \pm 0.14$ & & $<1$ \\
$\left|V_{u b}\right| \cdot 10^{3}(\mathrm{incl})$ & $4.19 \pm 0.20$ & & $\sim 2.2$ \\
\hline $\mathrm{BR}(B \rightarrow \tau v)\left[10^{-4}\right]$ & $1.09 \pm 0.24$ & $0.87 \pm 0.05$ & $<1$ \\
$A_{S L}^{d} \cdot 10^{3}$ & $-2.1 \pm 1.7$ & $-0.32 \pm 0.03$ & $<1$ \\
$A_{S L}^{s} \cdot 10^{3}$ & $-0.6 \pm 2.8$ & $0.014 \pm 0.001$ & $<1$ \\
\hline \hline
\end{tabular}

Special treatment is reserved to a few cases. Regarding the inputs coming from the semileptonic $B$ decays, the exclusive inputs are taken from the FLAG collaboration [5], while for the inclusive values we use different sources. For the $\left|V_{u b}\right|$ inclusive value we use the latest HFLAV determination 

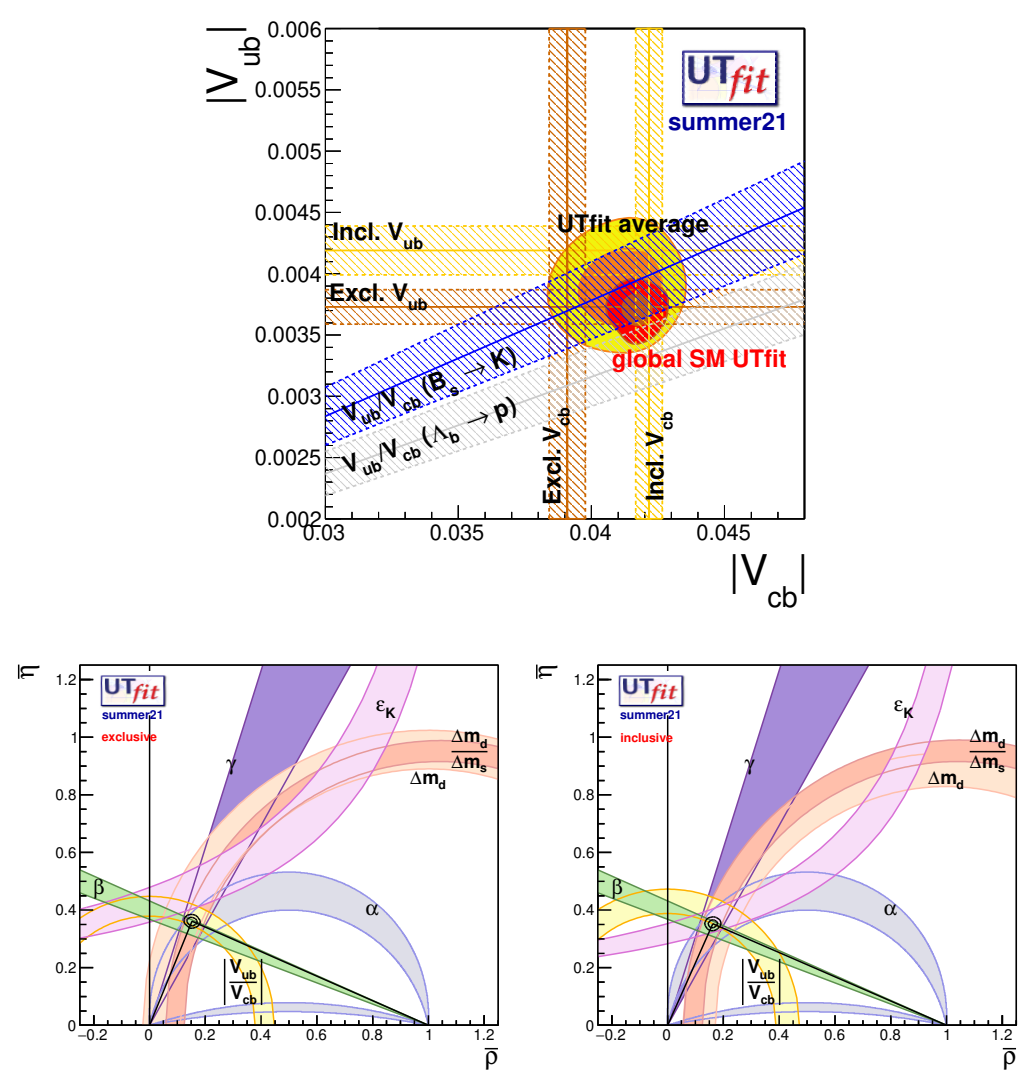

Figure 1: Top: $\left|V_{c b}\right|$ vs $\left|V_{u b}\right|$ plane showing the values reported in Table 1. We include in the average the LHCb ratio measurement [6] that is shown as a diagonal band. Bottom: $\bar{\rho}-\bar{\eta}$ plane with the SM global fit results using only exclusive inputs for both $V_{u b}$ and $V_{c b}$ (left) and using only inclusive inputs (right).
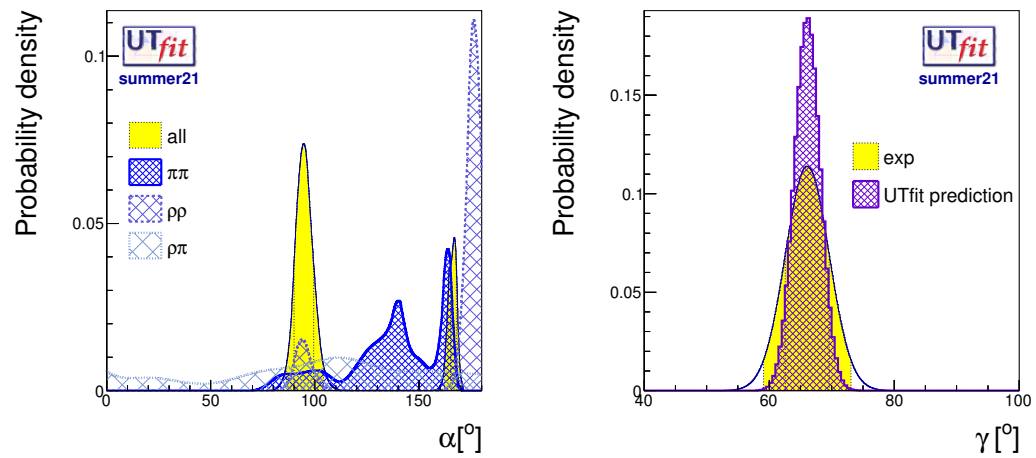

Figure 2: Left: global fit input distribution for the angle $\alpha$ (in solid yellow histogram) with the three separate distributions coming from the three contributing final states $\pi \pi, \rho \rho$ and $\rho \pi$. Right: global fit input distribution for the angle $\gamma$ (in solid yellow histogram) obtained by the HFLAV [3] average compared with the global UTfit prediction for the same angle.

obtained via the GGOU (Gambino, Giordano, Ossola and Uraltsev [7]) calculation, and then we add a flat uncertainty covering the spread of central values from the other calculations: this results 
in a value of $(4.19 \pm 0.17 \pm 0.18$ [flat] $) 10^{-3}$. For the $\left|V_{c b}\right|$ inclusive value we use the calculation from Ref. [8]. The values used are also shown in the left plot in Fig. 1: the UTfit two-dimensional (2D) average shown is calculated with a 2D procedure inspired by the skeptical method of Ref. [9] with $\sigma=1$. It is evident that exclusive and inclusive results persist in showing discrepancies in particular in the case of $\left|V_{c b}\right|$ at the level of about $3.6 \sigma$, while in the case of $V_{u b}$ it is reduced to about $1.8 \sigma$. The effect of these deviations in the global fit results are shown in the right plots in Fig. 1. These inclusive-vs-exclusive discrepancies have been highlighted and discussed by the UT fit collaboration since 2006 [10].

Regarding the angle inputs, the values used are as follows:

$\beta$ (or $\phi_{1}$ ): the value of $\sin 2 \beta$ is taken from the latest HFLAV average [3] using exclusively the $J / \psi K^{0}$ final states which give $\sin 2 \beta=0.698 \pm 0.017$. We then add a correction factor of $-0.01 \pm 0.01$ as data-driven theory uncertainty obtained with the method described in Ref. [11] with the most updated inputs.

$\alpha$ (or $\phi_{2}$ ): the value of the angle $\alpha$ is obtained by $\mathbf{U} f i t$ isospin analyses of the three contributing final states $\pi \pi, \rho \rho$ and $\rho \pi$. The various probability distributions are shown in Fig. 2 together with the combined one that is used as input to our global fit.

$\gamma\left(\right.$ or $\left.\phi_{3}\right)$ : the value of the angle $\gamma$ is taken from the latest HFLAV average [3] and the corresponding probability distribution is shown in Fig. 2 together with the prediction from the global fit.
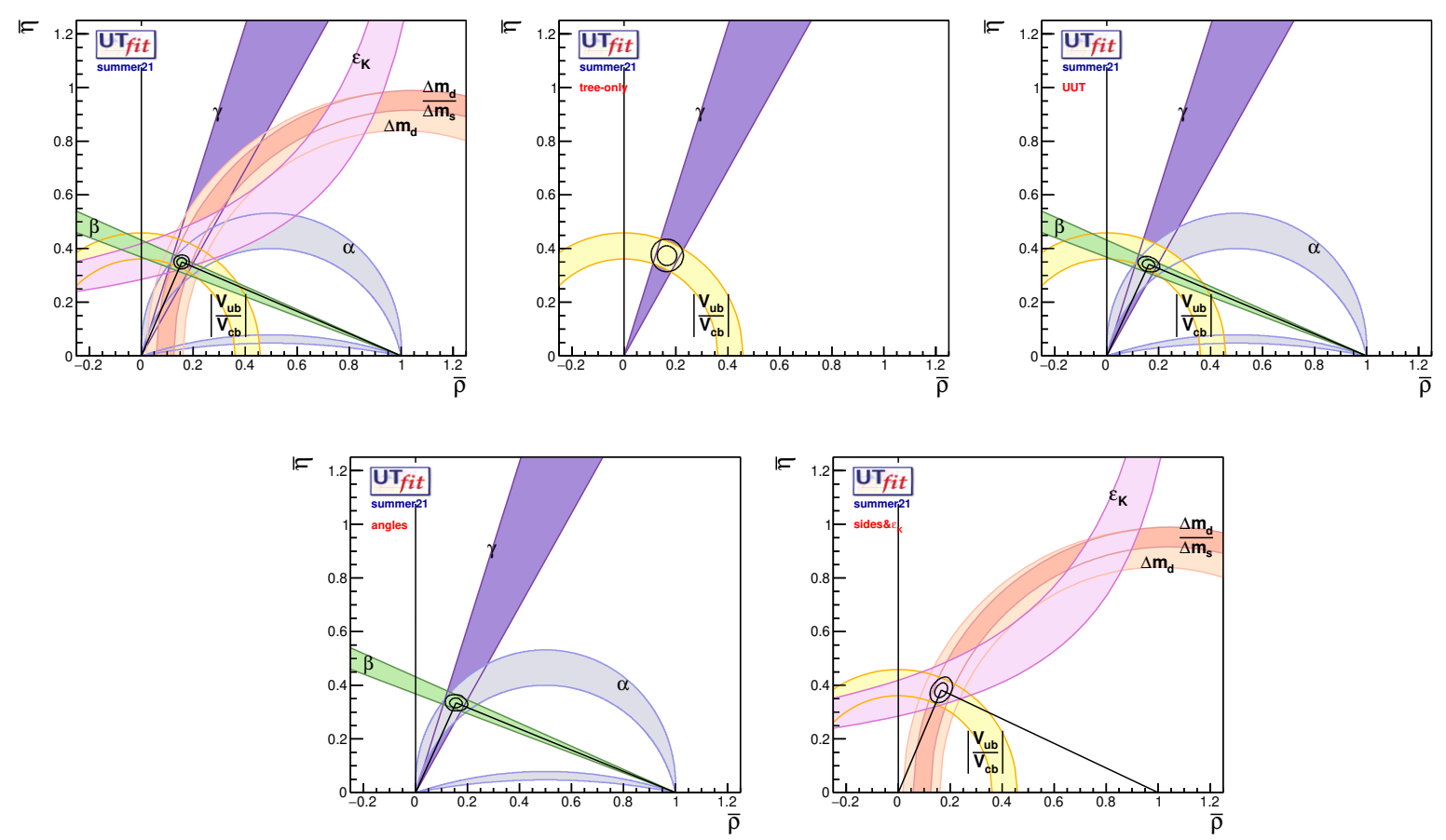

Figure 3: $\bar{\rho}-\bar{\eta}$ planes with the SM global fit results in various configurations. The black contours display the $68 \%$ and $95 \%$ probability regions selected by the given global fit. The $95 \%$ probability regions selected are also shown for each constraint considered. Top-Left: full SM fit; Top-Centre: fit using as inputs the "tree-only" constraints; Top-Right: Universal Unitarity Triangle fit; Bottom-Left: fit using as inputs only the angle measurements; Bottom-Right: fit using as inputs only the side measurements and the mixing parameter $\varepsilon_{K}$ in the kaon system. 

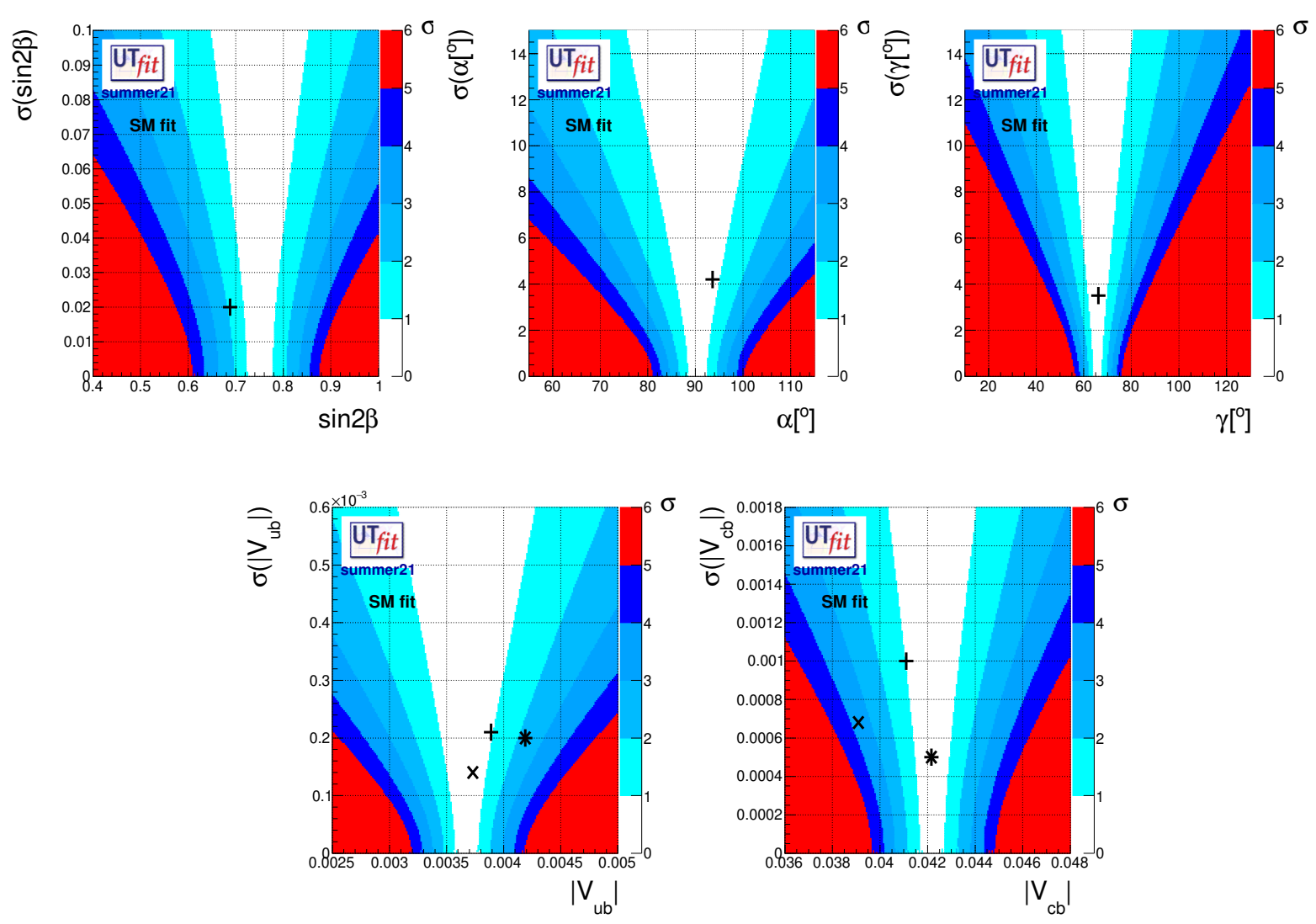

Figure 4: Pull plots (see text) for $\sin 2 \beta$ (top-left), $\alpha$ (top-centre), $\gamma$ (top-right), $\left|V_{u b}\right|$ (bottom-left) and $\left|V_{c b}\right|$ (bottom-right) inputs.

The results of the global SM fit are given as two-dimensional probability distributions in the plan of CKM parameters $\bar{\rho}$ and $\bar{\eta}$ and shown in Fig. 3. The numerical results are in Table 2.

Table 2: Results for the $\bar{\rho}$ and $\bar{\eta}$ values as extracted from the various fit configurations. The Universal Unitarity Triangle (UUT) fit includes the three angles inputs and the semileptonic $\left|V_{u b} / V_{c b}\right|$ [12].

\begin{tabular}{lcc}
\hline \hline fit configuration & $\bar{\rho}$ & $\bar{\eta}$ \\
\hline full SM fit & $0.156 \pm 0.012$ & $0.350 \pm 0.010$ \\
tree-only fit & $\pm 0.166 \pm 0.025$ & $\pm 0.374 \pm 0.025$ \\
UUT fit & $0.162 \pm 0.017$ & $0.341 \pm 0.011$ \\
angle-only fit & $0.156 \pm 0.017$ & $0.334 \pm 0.012$ \\
Sides $+\varepsilon_{K}$ fit & $0.166 \pm 0.018$ & $0.382 \pm 0.020$ \\
\hline
\end{tabular}

The so-called "pull plots" in Fig. 4 are used to assess the agreement of a given measurement with the indirect determination from the fit using all the other inputs. The coloured areas represent the level of agreement between the predicted values and the measurements at better than 1,2 , $\ldots n \sigma$. The markers have the coordinates $(x, y)=$ (central value, error) of the direct measurements considered. These plots allow to visualise the tensions between each input and the rest of them as in the pull column of Table 1. It is clear that inputs like $\alpha$ and $\gamma$ show perfect agreement wit the rest 
of the fit, while $\sin 2 \beta$ and $\left|V_{x b}\right|$ present various degrees of tension either directly or with respect to the different exclusive or inclusive determinations.

However, overall, the global fit proves a remarkable internal consistency with a better then $7 \%$ precision in the determination of the fundamental CKM parameters $\bar{\rho}$ and $\bar{\eta}$.

\section{References}

[1] N. Cabibbo, Unitary symmetry and leptonic decays, Phys. Rev. Lett. 10 (Jun, 1963) 531533; M. Kobayashi and T. Maskawa, CP Violation in the Renormalizable Theory of Weak Interaction, Prog.Theor.Phys. 49 (1973) 652-657.

[2] M. Ciuchini et al., 2000 CKM triangle analysis: A Critical review with updated experimental inputs and theoretical parameters, JHEP 0107 (2001) 013, [hep-ph/0012308]; UTFIT collaboration, M. Bona et al., The 2004 UTfit collaboration report on the status of the unitarity triangle in the standard model, JHEP 0507 (2005) 028, [hep-ph/0501199].

[3] HFLAV collaboration, Y. S. Amhis et al., Averages of b-hadron, c-hadron, and $\tau$-lepton properties as of 2018, Eur. Phys. J. C 81 (2021) 226, [1909. 12524].

[4] Particle Data Group collaboration, P. Zyla et al., Review of Particle Physics, PTEP 2020 (2020) 083 C01.

[5] Flavour Lattice Averaging Group collaboration, S. Aoki et al., FLAG Review 2019: Flavour Lattice Averaging Group (FLAG), Eur. Phys. J. C 80 (2020) 113, [1902 . 08191].

[6] LHCв collaboration, R. Aaij et al., Determination of the quark coupling strength $\left|V_{u b}\right|$ using baryonic decays, Nature Phys. 11 (2015) 743-747, [1504.01568].

[7] P. Gambino, P. Giordano, G. Ossola and N. Uraltsev, Inclusive semileptonic B decays and the determination of $|V(u b)|$, JHEP 10 (2007) 058, [0707 . 2493].

[8] M. Bordone, B. Capdevila and P. Gambino, Three loop calculations and inclusive Vcb, Phys. Lett. B 822 (2021) 136679, [2107.00604].

[9] G. D'Agostini, Sceptical combination of experimental results: General considerations and application to epsilon-prime / epsilon, Submitted to: Phys. Rev. D (1999), [hep-ex/9910036].

[10] UTFit collaboration, M. Bona et al., The Unitarity Triangle Fit in the Standard Model and Hadronic Parameters from Lattice QCD: A Reappraisal after the Measurements of $\Delta m_{s}$ and $B R\left(B \rightarrow \tau v_{\tau}\right)$, JHEP 0610 (2006) 081, [hep-ph/0606167].

[11] M. Ciuchini, M. Pierini and L. Silvestrini, The Effect of penguins in the $B(d) \rightarrow J /$ psi KO CP asymmetry, Phys. Rev. Lett. 95 (2005) 221804, [hep-ph/0507290].

[12] A. J. Buras, P. Gambino, M. Gorbahn, S. Jager and L. Silvestrini, Universal unitarity triangle and physics beyond the standard model, Phys. Lett. B 500 (2001) 161-167, [hep-ph/0007085]. 